\title{
Comparing Gamma-ray Loud and Gamma-ray Quiet Radio Pulsars - A Unification Scheme
}

\author{
Patrick Weltevrede ${ }^{1}$ \\ ${ }^{1}$ Jodrell Bank Centre for Astrophysics, The University of Manchester \\ Alan Turing Building, Manchester, M13 9PL, United Kingdom \\ email: Patrick. Weltevrede@manchester.ac.uk
}

\begin{abstract}
A radio polarization study of gamma-ray-detected pulsars reveals a surprising tendency for the magnetic and rotation axes to be relatively aligned. This provides tension with gamma-ray models, which disfavour such alignment. The lack of correlation between these findings and those derived from the gamma-ray light curves suggests problems in the models. To make the data consistent with a random orientation of the magnetic field the emission regions could be assumed to extend outside what is traditionally thought to be the open-field-line region in a magnetic inclination angle dependent way. Both acceptance and rejection of this hypothesis has important consequences. Finally, a unification scheme is proposed to explain the observational differences between gamma-ray loud and gamma-ray quiet radio pulsars. This unification scheme takes the orientation of the line of sight and the magnetic inclination angle to be key parameters affecting both the radio and gamma-ray light-curve morphology.
\end{abstract}

Keywords. pulsars: general, gamma rays: observations

\section{The radio polarization and gamma-rays of young pulsars}

In a series of papers (Rookyard et al. 2015a, 2015b \& 2017) the radio and gamma-ray properties of young energetic pulsars were explored. The aim was to study the geometry of the pulsar magnetospheres exploiting radio polarization techniques, and compare the radio properties of gamma-ray loud pulsars with their gamma-ray quiet counterparts.

\subsection{The radio polarization of gamma-ray loud pulsars}

A sample of 28 pulsars was selected which are regularly monitored by Parkes (Weltevrede et al. 2010) and which appear in the second Fermi Large Area Telescope catalog of gamma-ray pulsars (Abdo et al. 2013). In contrast to the general pulsar population, these pulsars show position angles of the linear polarization that depend on the rotational phase of the neutron star which are well described with the Rotating Vector Model. Together with the measured radio pulse width and considering aberration and retardation effects this leads to constraints on the viewing geometry. These constraints were derived by Rookyard et al. (2015a) after carefully considering different sources of statistical and systematic errors using a novel technique. The used software for fitting and visualisation are freely available within the PSRSalsa package (Weltevrede 2016, see also http://www.jb.man.ac.uk/ pulsar/Resources/psrsalsa.html).

The analysis resulted in a number of surprizes. First of all there are many pulsars in the sample which have relatively low magnetic inclination angles $\alpha$. The left panel of figure 1 shows the observed $\alpha$ distribution compared to the distribution expected when the magnetic angle distribution is completely random (dashed line). This could be interpreted to mean that during the pulsar formation process an aligned magnetic axis 

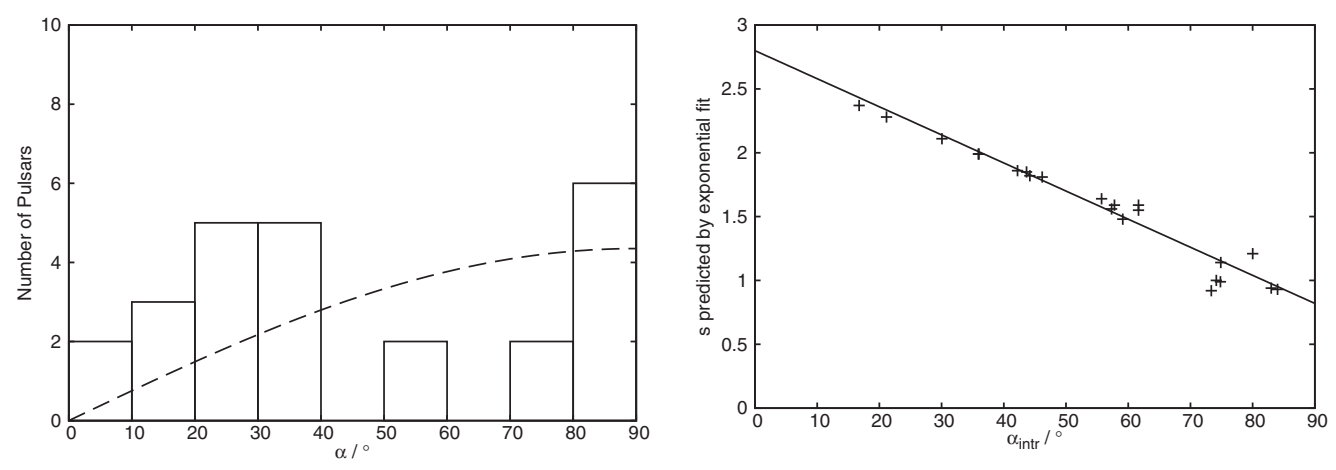

Figure 1. Left panel: Distribution of derived $\alpha$ values for a sample of young pulsars compared to a random distribution which might be expected for a sample of young pulsars (dashed curve). Right panel: The $\alpha$ distribution can be made consistent with a random distribution when "extra-cap" emission is allowed: emission from beyond what is traditionally is thought to be the open field line region. The more aligned the magnetic axis is with the rotation axis, the stronger the deviation. An aligned rotator is required to have a radio beam which is more than 2.5 times wider than one might simplistically expect. Figures from Rookyard et al. (2015a \& 2015b).

is favoured, or a low $\alpha$ makes it more likely that significant radio emission is produced. This conclusion is reinforced by the fact that there are important biases to consider that are expected to skew the observed $\alpha$ distribution in the opposite direction. First of all, a low $\alpha$ implies a low beaming fraction, i.e. a low probability for our line of sight to coincide with the radio beam. Secondly, the considered sample is selected based on the fact that pulsed gamma-rays are observed, which is expected to be more difficult when $\alpha$ is low. Although alignment (secular evolution towards low $\alpha$ ) has been suggested from observations (e.g. Weltevrede \& Johnston 2008), the associated timescale is far too long to be relevant for this young sample of pulsars.

To avoid the conclusion that neutron stars are more likely to form with low magnetic inclination angles, it would be necessary to relax one or more assumptions which are typically made when deriving viewing geometries from radio data. An extensive range of scenario's was explored by Rookyard et al. (2015b) who concluded that the most likely scenario, under the assumption that pulsars are born with a random magnetic inclination angle distribution, is that the radio beam is larger than expected from simple magnetic dipole geometry when $\alpha$ is low. Although this could imply that radio emission is produced on closed field lines, it could also imply that the open field line region is significantly wider at the radio emission height for these pulsars (see also e.g. Weltevrede \& Wright 2009). The deviation from simple textbook physics disappears for orthogonal rotators, which might be of theoretical significance. Under the assumption that neutron stars are born with random magnetic inclination angles, a simple correction scheme for the radio derived geometric parameters exists (Rookyard et al. 2015b), independent from the underlying physical reason for the deviation.

A second surprize when deriving viewing geometries from the gamma-ray detected radio pulsars was that the measured $\alpha$ values from the radio data are inconsistent with those derived from the shape of the gamma-ray light curves (Rookyard et al. 2015a). No apparent correlation was found, irrespective of what underlying gamma-ray model was assumed. This is potentially very worrying, since it suggests that we do not understand how to interpret the radio and/or the gamma-ray data. However, subsequent analysis revealed that at least qualitatively the basic geometrical picture of how radio and 
gamma-rays are produced in pulsars appears to be correct and that the magnetic inclination angle plays a crucial role.

\subsection{Comparison of the emission properties of gamma-ray loud $\mathcal{G}$ quiet radio pulsars}

The aim of a study by Rookyard et al. (2017) was to see if there are observed correlations between radio and gamma-ray pulse profile morphologies. In addition the aim was to qualitatively understand why some pulsars with a high spin-down luminosity are gammaray detected, while others are not (apart from distance). Here the most important results from this analysis are summarized and interpreted in the framework of the existing models, with the key geometric parameter being $\alpha$.

From the top panel of figure 2 it is apparent that gamma-ray detected radio pulsars tend to have narrow radio profiles (the open and closed symbols are roughly separated by the dashed line). This is exactly what you would expect from theory, since it is believed that gamma-rays are preferentially produced in the direction of the rotational equator. This implies that in order to detect the gamma-rays, the line of sight will be preferentially in the direction of the rotational equator. To detect the radio as well as the gamma-rays, the radio beam (hence the magnetic axis) need to be sufficiently inclined. This typically result in narrower radio profiles. Note that this correlation suggests that geometrical parameters are very important for both the radio and gamma-rays pulse profile morphologies.

The bottom panel of figure 2 (ignoring the points at $\Delta=0$ for the moment) suggests that there is an anti-correlation between the radio and gamma-ray pulse widths (i.e. the points roughly follow the solid line). A generic feature of caustic emission being responsible for the gamma-ray light curve morphologies is that double-peaked light curves should have a large peak separation for highly inclined rotators (e.g. Watters et al. 2009), while highly inclined rotators indeed should produce narrow radio profiles.

Finally, highly inclined pulsars should have sharper light-curve components (e.g. Watters et al. 2009). Therefore gamma-ray pulsars with sharp components (open symbols in the bottom panel of figure 2) should have narrow radio profiles. Indeed the open and closed symbols can be roughly separated by the dashed line.

\section{Conclusions}

Striking (model-independent) correlations between the radio and gamma-ray light curve morphologies have been found. The results are consistent with the unifying picture where all high spin-down luminosity pulsars produce gamma-rays, but there are geometrical reasons which determine their gamma-ray detectability. The magnetic inclination angle $\alpha$ appears to be the key parameter to explain the results, while other factors (such as distance) will weaken the found correlations.

The fact that the unification model works implies that the discussed tendency of young gamma-ray detected pulsars to have low inclination angles even more curious, as it implies an opposite bias should exist. This conclusion will have consequences, for example, for population synthesis studies as the inclination angle affect the beaming fraction. A remaining open question is if the results imply that pulsars are typically born with an aligned magnetic axis, or that the radio and/or gamma-ray models need to be quantitatively modified. Rookyard et al. (2017) suggest that emission from beyond what is traditionally thought to be the open field line region could be an alternative to the preferential formation of aligned pulsars hypothesis. 

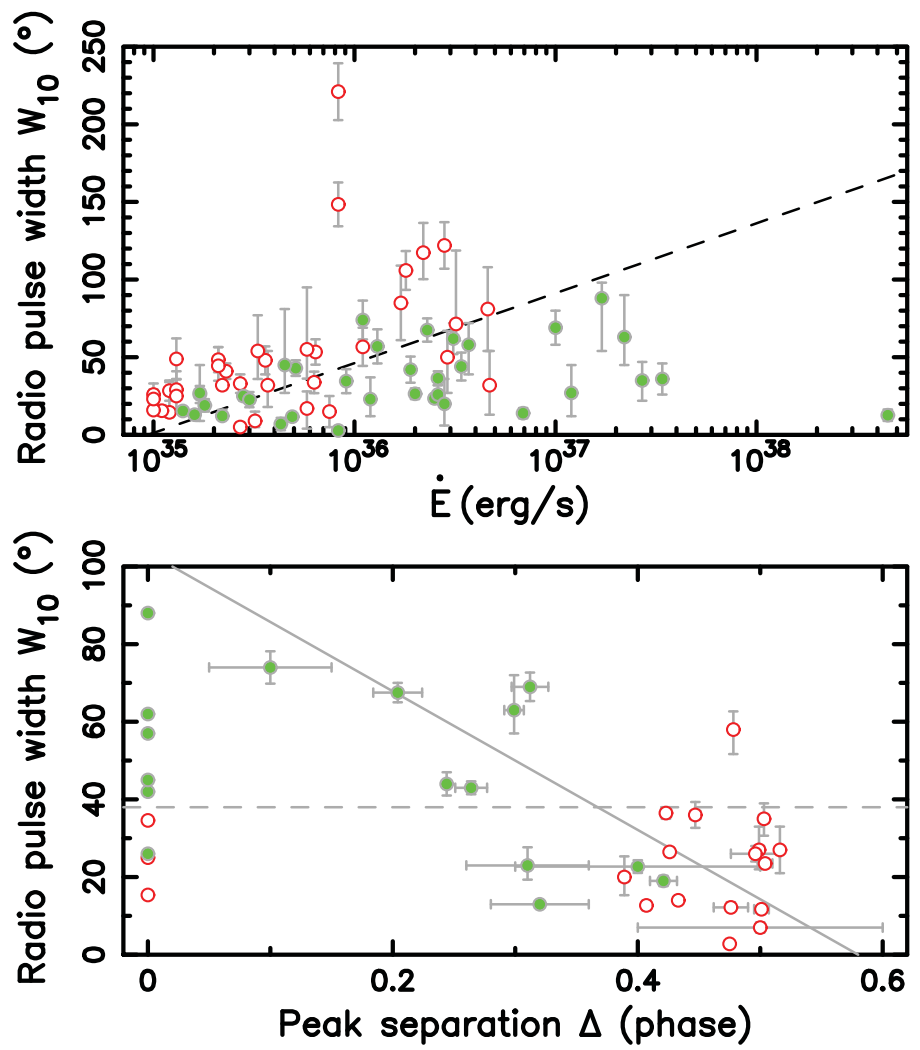

Figure 2. Top panel: The measured radio pulse width (at $10 \%$ of the peak intensity) as function of spin-down energy loss rate. The filled symbols represent those pulsars which are gamma-ray detected according to the second Fermi Large Area Telescope catalog of gamma-ray pulsars (Abdo et al. 2013). Note that many gamma-ray detected pulsars are below the dashed line, indicating that they typically have a narrower radio profiles compared to their non gamma-ray detected counterparts. Bottom panel: The measured radio pulse width of gamma-ray detected pulsars as function of the peak separation of double-peaked gamma-ray light curves. Those pulsars which do not have a double-peaked light curve are assigned $\Delta=0$. As indicated by the solid line, there is an anti-correlation between the width of the radio and gamma-ray profiles. The sharpest gamma-ray light curves are indicated as open symbols. Note that the corresponding radio profiles are relatively narrow, as indicated by the horizontal dashed line. Figures adapted from Rookyard et al. (2017).

\section{References}

Abdo, A. A., et al. 2013, ApJS, 208, 17

Rookyard, S. C., Weltevrede, P., \& Johnston, S. 2015a, MNRAS, 446, 3367

Rookyard, S. C., Weltevrede, P., \& Johnston, S. 2015b, MNRAS, 446, 3356

Rookyard, S. C., Weltevrede, P., Johnston, S., \& Kerr, M. 2017, MNRAS, 464, 2018

Watters, K. P., Romani, R. W., Weltevrede, P., \& Johnston, S. 2009, ApJ, 694, 1289

Weltevrede, P. 2016, A\&A A, 590, 109

Weltevrede, P., et al. 2010, Publications of the Astron. Soc. Australia, 27, 64

Weltevrede, P. \& Johnston, S. 2008, MNRAS, 387, 1755

Weltevrede, P. \& Wright, G. 2009, MNRAS, 395, 2117 